\title{
Aspectos Biométricos de la Mano de Individuos Chilenos
}

\author{
Biometric Aspects of the Hand in Chilean Individuals
}

*Binvignat, O. **Almagià, A.; **Lizana, P.; \& **** Olave, E.

\author{
BINVIGNAT, O; ALMAGIÀ, A.; LIZANA, P. \& OLAVE, E. Aspectos biométricos de la mano de individuos chilenos. Int. J. Morphol., \\ 30(2):599-606, 2012.
}

\begin{abstract}
RESUMEN: La mano es fundamental para ejecutar diversas acciones tanto en el campo laboral como en la entretención y el deporte, pudiendo realizar funciones muy básicas hasta otras muy especializadas. La función de la mano se puede analizar mejor cuando es dividida en partes, aunque es algo meramente didáctico y artificial, permitiendo una mejor comprensión en la actividad de la mano como una unidad. Debido a la importancia de esta parte del cuerpo y a la escasa información de parámetros biométricos que existe en nuestra población, se ha tornado necesario un estudio acabado sobre las dimensiones de la mano como un todo, así como también de sus componentes, los dedos. De acuerdo a ello, se analizaron 1.196 manos pertenecientes a habitantes de ambos sexos (267 hombres y 331 mujeres) de edad entre 18 y 65 años, nacidos y residentes en la VII región de Chile -Maule. Cada paciente fue sometido a un examen antropométrico general y uno específico de la mano. Las variables consideradas fueron las siguientes: longitud de la mano, ancho de la mano, longitud de los dedos, longitud del sector de las falanges. Las variables se estudiaron de acuerdo a sexo, edad y lados. Parte de los resultados fueron los siguientes: en el sexo masculino, la longitud promedio de la mano derecha fue de 184,9 mm y de la izquierda de 185,6; en el sexo femenino fue de 167,6 mm en el lado derecho y de 168,9 en el izquierdo. Con respecto a la longitud de los dedos en el sexo masculino, considerando la sumatoria de los promedios obtenidos para cada sector de la falanges correspondientes, los promedios fueron: pulgar, 72,5 mm en la mano derecha y 72,4 mm en la izquierda; indicador, 104,2 y 104,5 mm; medio, 115 y 114,6 mm; anular, 109,1 y $108,9 \mathrm{~mm}$ y el mínimo, 88,5 y 88,4 mm, respectivamente. En el sexo femenino, la longitudes promedios fueron: pulgar de la mano derecha, 63,5 mm y 62,6 mm en la izquierda; indicador, 91 y 90,7 mm; medio, 100,4 y 99,2 mm; anular, 94,4 y 94,2 mm y el mínimo, 75,8 y 75,9 mm, respectivamente. Los datos aportados en este trabajo son de importancia, ya que permiten tener un conocimiento real de variables biométricas de la mano en individuos chilenos.
\end{abstract}

PALABRAS CLAVE: Mano; Dedos; Falanges Biometría.

\section{INTRODUCCIÓN}

La mano es fundamental para ejecutar diversas acciones tanto en el campo laboral como en la entretención y el deporte, pudiendo realizar funciones muy básicas hasta otras muy especializadas. La utilización de las manos en el desarrollo de actividades tan cotidianas como trabajar, estudiar o simplemente ejecutar quehaceres domésticos, pueden afectar directamente nuestra vida diaria, ya que la constante actividad que desarrollan las manos puede llevar a tener lesiones muy dolorosas, que a veces, pueden producir pérdida de su función temporal y parcialmente o ser crónica $\mathrm{y}$ afectar al individuo por toda su vida.

A través de la mano así como a través de la visión y audición, se puede formar una concepción del mundo externo, siendo como una extensión de nuestro cerebro en el mundo circundante. La función de la mano se puede analizar mejor cuando es dividida en partes, aunque es algo me- ramente didáctico y artificial, permitiendo una mejor comprensión en la actividad de la mano como una unidad. Los rasgos más característicos de la mano humana son la longitud comparativa del dedo pulgar y sus músculos asociados, extrínsecos e intrínsecos, que le permiten gran flexibilidad y eficacia junto con el resto de la mano. La oposición del dedo pulgar en el hombre permite una gama de actividades muy especializadas.

Siendo tan importante y por ende, muy utilizada en labores diarias, se expone a una gama de lesiones que pueden afectar su función, teniendo como resultado, muchas veces, incapacidades que conllevan a un individuo a tener serios problemas en su vida futura.

Debido a la importancia de esta parte del cuerpo y a la escasa información de parámetros biométricos que existe

* Universidad Autónoma de Chile, Sede Talca.

** Pontificia Universidad Católica de Valparaíso, Chile.

**** Universidad de La Frontera, Temuco, Chile. 
en nuestra población, se realizó un estudio acabado sobre las dimensiones de la mano como un todo, así como también de sus componentes, los dedos, comparando estos datos con grupos humanos de otras latitudes.

\section{MATERIAL Y MÉTODO}

Se analizaron 1.196 manos pertenecientes a habitantes de ambos sexos (267 hombres y 331 mujeres), de edad entre 18 y 65 años, nacidos y residentes en la VII región de Chile -Maule. Cada persona fue sometida a un examen antropométrico general y uno específico de la mano, contando previamente con la aceptación voluntaria de participar en esta investigación. Además de las mediciones antropométricas específicas, se obtuvieron fotografías digitales de las manos, para una segunda evaluación métrica computarizada (software Carnoy). Las variables consideradas fueron las siguientes: longitud de la mano, ancho de la mano, longitud de los dedos y longitud del sector de las falanges. Las variables se estudiaron de acuerdo a sexo, edad, talla y lados.

El registro de la longitud de la mano se consideró desde el pliegue distal de la muñeca hasta el extremo distal del dedo medio; el ancho de la mano se registró desde el margen lateral de la palma, antes del pliegue digito palmar del dedo indicador, hasta el margen medial de la palma, previo al pliegue digito palmar del dedo mínimo; la longitud de los dedos se midió entre la articulación metacarpofalángica, por su cara dorsal, hasta el extremo distal de los dedos; la longitud de la zona correspondiente a las falanges se midieron por la cara dorsal de la mano; la de la falange proximal se registró entre la articulación metacarpofalángica y la interfalángica proximal; la de la falange media, entre la articulación interfalángica proximal y la distal, y, la de la falange distal entre la articulación interfalángica distal y el extremo de los dedos. Para el registro de las medidas se utilizó un calyper Mitutoyo de 0,05 mm de precisión.

\section{RESULTADOS}

La edad promedio de los individuos de sexo masculino fue de 32 años, un peso de $77 \mathrm{~kg}$ y $170 \mathrm{~cm}$ de estatura: la edad promedio en el sexo femenino fue de 33 años, $77 \mathrm{~kg}$ de peso y $165 \mathrm{~cm}$ de estatura.

Los promedios obtenidos por sexo, lados derecho e izquierdo y grupos etarios para la longitud y ancho de la mano, así como de la longitud de la palma se muestran en la Tablas I - XII.

Con respecto a la longitud de los dedos en hombres, considerando la sumatoria de los promedios obtenidos para cada sector de la falanges correspondientes, los promedios fueron los siguientes: pulgar, 72,5 $\mathrm{mm}$ en la mano derecha y $72,4 \mathrm{~mm}$ en la mano izquierda; indicador, 104,2 y 104,5 mm; medio, 115 y 114,6 mm; anular, 109,1 y 108,9 mm y el mínimo, 88,5 y 88,4 mm, respectivamente.

En mujeres, las longitudes promedios fueron: pulgar de la mano derecha, $63,5 \mathrm{~mm}$ y $62,6 \mathrm{~mm}$ en la mano izquierda; indicador, 91 y 90,7 mm; medio, 100,4 y 99,2 mm; anular, 94,4 y $94,2 \mathrm{~mm}$ y el mínimo, 75,8 y $75,9 \mathrm{~mm}$, respectivamente.

Tabla I. Promedios de la longitudes, anchura de la mano y longitud de la palma en mujeres expresado en mm.

\begin{tabular}{lllllll}
\hline & Longitud & mano & \multicolumn{1}{c}{ Ancho } & mano & Longitud & Palma \\
\hline edades & derecha & izquierda & derecha & izquierda & derecha & izquierda \\
\hline $18-19$ & $166 \pm 25$ & $169 \pm 10$ & $81 \pm 13$ & $82 \pm 17$ & $92 \pm 8$ & $94 \pm 8$ \\
$20-24$ & $166 \pm 32$ & $173 \pm 10$ & $78 \pm 10$ & $77 \pm 9$ & $95 \pm 8$ & $95 \pm 9$ \\
$25-29$ & $171+5$ & $171+5$ & $81+18$ & $80+19$ & $92+8$ & $91+11$ \\
$30-34$ & $170+11$ & $168+10$ & $82+17$ & $80+18$ & $96+10$ & $96+10$ \\
$35-39$ & $172+6$ & $172+7$ & $80+9$ & $78+6$ & $95+6$ & $95+6$ \\
$40-44$ & $170 \pm 8$ & $169 \pm 8$ & $80 \pm 7$ & $79 \pm 7$ & $97 \pm 4$ & $96 \pm 4$ \\
$45-49$ & $165 \pm 10$ & $166 \pm 10$ & $78 \pm 9$ & $78 \pm 8$ & $95 \pm 6$ & $96 \pm 7$ \\
$50-54$ & $169 \pm 11$ & $171 \pm 10$ & $80 \pm 7$ & $80 \pm 7$ & $98 \pm 19$ & $97 \pm 15$ \\
$55-59$ & $166 \pm 11$ & $166 \pm 11$ & $78 \pm 8$ & $77 \pm 8$ & $90 \pm 11$ & $90 \pm 11$ \\
$60-64$ & $158 \pm 7$ & $160 \pm 10$ & $76 \pm 7$ & $74 \pm 7$ & $83 \pm 12$ & $86 \pm 13$ \\
$65-69$ & $171+8$ & $172+9$ & $76+5$ & $77+8$ & $96+8$ & $97+8$
\end{tabular}


BINVigNAT, O; ALMAGiÀ, A.; LIZANA, P. \& OLAVE, E. Aspectos biométricos de la mano de individuos chilenos. Int. J. Morphol., 30(2):599-606, 2012.

Tabla II. Promedios de la longitudes, anchura de la mano y longitud de la palma, en hombres expresado en mm.

\begin{tabular}{lllllll}
\hline & Longitud & mano & Ancho & mano & Longitud & Palma \\
\hline edades & derecha & izquierda & derecha & izquierda & derecha & izquierda \\
\hline $18-19$ & $182 \pm 10$ & $183 \pm 10$ & $95 \pm 19$ & $94 \pm 19$ & $102 \pm 8$ & $103 \pm 7$ \\
$20-24$ & $186 \pm 9$ & $188 \pm 11$ & $92 \pm 19$ & $91 \pm 19$ & $105 \pm 7$ & $104 \pm 7$ \\
$25-29$ & $188 \pm 10$ & $188 \pm 10$ & $92 \pm 21$ & $90 \pm 21$ & $105 \pm 8$ & $109 \pm 12$ \\
$30-34$ & $183 \pm 12$ & $186 \pm 12$ & $89 \pm 10$ & $89 \pm 9$ & $104 \pm 9$ & $106 \pm 8$ \\
$35-39$ & $184 \pm 8$ & $183 \pm 9$ & $97 \pm 13$ & $96 \pm 14$ & $101 \pm 8$ & $109 \pm 27$ \\
$40-44$ & $184 \pm 15$ & $184 \pm 15$ & $89 \pm 10$ & $88 \pm 8$ & $106 \pm 15$ & $106 \pm 13$ \\
$45-49$ & $183 \pm 12$ & $184 \pm 6$ & $91 \pm 8$ & $99 \pm 6$ & $106 \pm 11$ & $102 \pm 7$ \\
$50-54$ & $186 \pm 13$ & $186 \pm 13$ & $99 \pm 0$ & $91 \pm 13$ & $109 \pm 18$ & $107 \pm 13$ \\
$55-59$ & $187 \pm 16$ & $187 \pm 20$ & $92 \pm 13$ & $87 \pm 4$ & $113 \pm 25$ & $105 \pm 8$ \\
$60-64$ & $186 \pm 12$ & $187 \pm 12$ & $87 \pm 4$ & & & $106 \pm 10$ \\
\hline
\end{tabular}

Tabla III. Longitudes promedio del sector de las falanges del dedo pulgar en mujeres, expresado en $\mathrm{mm}$.

\begin{tabular}{lllll}
\hline & Falange & proximal & Falange & distal \\
\hline edades & Mano der & Mano izq & Mano der & Mano izq \\
\hline $18-19$ & $36 \pm 8$ & $37 \pm 9$ & $29 \pm 4$ & $28 \pm 4$ \\
$20-24$ & $37 \pm 6$ & $36 \pm 7$ & $29 \pm 4$ & $28 \pm 4$ \\
$25-29$ & $38 \pm 5$ & $37 \pm 7$ & $29 \pm 3$ & $29 \pm 3$ \\
$30-34$ & $32 \pm 7$ & $32 \pm 8$ & $27 \pm 5$ & $26 \pm 5$ \\
$35-39$ & $37 \pm 7$ & $36 \pm 6$ & $27 \pm 5$ & $27 \pm 4$ \\
$40-44$ & $35 \pm 6$ & $36 \pm 6$ & $28 \pm 3$ & $28 \pm 3$ \\
$45-49$ & $36 \pm 7$ & $35 \pm 7$ & $28 \pm 5$ & $27 \pm 4$ \\
$50-54$ & $35 \pm 7$ & $35 \pm 6$ & $29 \pm 6$ & $28 \pm 4$ \\
$55-59$ & $33 \pm 7$ & $33 \pm 7$ & $28 \pm 4$ & $28 \pm 4$ \\
$60-64$ & $34 \pm 8$ & $31 \pm 8$ & $28 \pm 3$ & $28 \pm 3$ \\
$65-69$ & $33 \pm 7$ & $35 \pm 8$ & $30 \pm 5$ & $29 \pm 2$ \\
\hline
\end{tabular}

Tabla IV. Longitudes promedio del sector de las falanges del dedo pulgar en hombres, expresado en mm.

\begin{tabular}{lllll}
\hline & Falange & proximal & Falange & distal \\
\hline edades & Mano der & Mano izq & Mano der & Mano izq \\
\hline $18-19$ & $41 \pm 7$ & $41 \pm 7$ & $32 \pm 4$ & $32 \pm 4$ \\
$20-24$ & $40 \pm 6$ & $40 \pm 6$ & $32 \pm 3$ & $32 \pm 3$ \\
$25-29$ & $42 \pm 7$ & $42 \pm 7$ & $34 \pm 4$ & $34 \pm 5$ \\
$30-34$ & $41 \pm 9$ & $41 \pm 8$ & $32 \pm 5$ & $31 \pm 5$ \\
$35-39$ & $39 \pm 8$ & $38 \pm 8$ & $32 \pm 4$ & $31 \pm 5$ \\
$40-44$ & $40 \pm 10$ & $40 \pm 11$ & $31 \pm 9$ & $31 \pm 8$ \\
$45-49$ & $44 \pm 4$ & $43 \pm 6$ & $33 \pm 3$ & $32 \pm 4$ \\
$50-54$ & $41 \pm 7$ & $40 \pm 7$ & $32 \pm 4$ & $32 \pm 4$ \\
$55-59$ & $39 \pm 11$ & $40 \pm 12$ & $33 \pm 4$ & $33 \pm 4$ \\
$60-64$ & $38 \pm 9$ & $38 \pm 10$ & $33 \pm 4$ & $33 \pm 5$ \\
\hline
\end{tabular}


Tabla V. Longitudes promedio del sector de las falanges del dedo indicador de mujeres, expresado en mm.

\begin{tabular}{|c|c|c|c|c|c|c|}
\hline & Falange & proximal & Falange & media & Falange & distal \\
\hline edades & Mano der & Mano izq & Mano der & Mano izq & Mano der & Mano izq \\
\hline $18-19$ & $44 \pm 12$ & $43 \pm 12$ & $27 \pm 7$ & $28 \pm 6$ & $22 \pm 3$ & $22 \pm 3$ \\
\hline $20-24$ & $45 \pm 11$ & $45 \pm 11$ & $28 \pm 6$ & $28 \pm 6$ & $22 \pm 3$ & $22 \pm 3$ \\
\hline $25-29$ & $46 \pm 11$ & $46 \pm 11$ & $29 \pm 5$ & $29 \pm 6$ & $23 \pm 2$ & $23 \pm 2$ \\
\hline $30-34$ & $36 \pm 15$ & $35 \pm 14$ & $24 \pm 7$ & $23 \pm 7$ & $20 \pm 4$ & $20 \pm 4$ \\
\hline $35-39$ & $44 \pm 11$ & $44 \pm 11$ & $28 \pm 6$ & $29 \pm 7$ & $21 \pm 4$ & $21 \pm 4$ \\
\hline $40-44$ & $46 \pm 11$ & $45 \pm 10$ & $28 \pm 6$ & $28 \pm 6$ & $22 \pm 2$ & $22 \pm 2$ \\
\hline $45-49$ & $45 \pm 10$ & $45 \pm 10$ & $28 \pm 6$ & $28 \pm 6$ & $23 \pm 3$ & $22 \pm 3$ \\
\hline $50-54$ & $43 \pm 13$ & $42 \pm 13$ & $27 \pm 6$ & $27 \pm 6$ & $22 \pm 3$ & $22 \pm 2$ \\
\hline $55-59$ & $38 \pm 15$ & $39 \pm 13$ & $25 \pm 8$ & $24 \pm 7$ & $22 \pm 4$ & $22 \pm 4$ \\
\hline $60-64$ & $37 \pm 12$ & $39 \pm 17$ & $25 \pm 7$ & $25 \pm 6$ & $21 \pm 3$ & $21 \pm 2$ \\
\hline $65-69$ & $40 \pm 16$ & $40 \pm 17$ & $27 \pm 9$ & $27 \pm 9$ & $23 \pm 3$ & $22 \pm 3$ \\
\hline
\end{tabular}

Tabla VI. Longitudes promedio del sector de las falanges del dedo indicador de hombres, expresado en mm.

\begin{tabular}{|c|c|c|c|c|c|c|}
\hline & Falange & proximal & Falange & media & Falange & distal \\
\hline edades & Mano der & Mano izq & Mano der & Mano izq & Mano der & Mano izq \\
\hline 18-19 & $51 \pm 12$ & $51 \pm 11$ & $31 \pm 6$ & $32 \pm 5$ & $24 \pm 3$ & $24 \pm 4$ \\
\hline $20-24$ & $50 \pm 12$ & $50 \pm 12$ & $31 \pm 6$ & $31 \pm 6$ & $24 \pm 3$ & $24 \pm 3$ \\
\hline $25-29$ & $50 \pm 13$ & $51 \pm 13$ & $31 \pm 7$ & $31 \pm 7$ & $25 \pm 3$ & $25 \pm 4$ \\
\hline $30-34$ & $49 \pm 12$ & $50 \pm 11$ & $32 \pm 7$ & $32 \pm 6$ & $25 \pm 4$ & $24 \pm 4$ \\
\hline $35-39$ & $43 \pm 13$ & $44 \pm 12$ & $30 \pm 6$ & $29 \pm 7$ & $24 \pm 3$ & $25 \pm 2$ \\
\hline $40-44$ & $50 \pm 16$ & $50 \pm 14$ & $30 \pm 9$ & $31 \pm 9$ & $24 \pm 6$ & $24 \pm 6$ \\
\hline $45-49$ & $53 \pm 12$ & $50 \pm 11$ & $32 \pm 7$ & $32 \pm 7$ & $24 \pm 3$ & $25 \pm 3$ \\
\hline $50-54$ & $51 \pm 11$ & $51 \pm 11$ & $31 \pm 6$ & $31 \pm 6$ & $24 \pm 3$ & $24 \pm 3$ \\
\hline $55-59$ & $47 \pm 16$ & $48 \pm 15$ & $30 \pm 9$ & $30 \pm 8$ & $25 \pm 3$ & $25 \pm 3$ \\
\hline $60-64$ & $45 \pm 16$ & $45 \pm 17$ & $31 \pm 9$ & $31 \pm 9$ & $25 \pm 3$ & $25 \pm 3$ \\
\hline
\end{tabular}

Tabla VII. Longitudes promedio del sector de las falanges del dedo medio de mujeres, expresado en mm.

\begin{tabular}{|c|c|c|c|c|c|c|}
\hline & Falange & proximal & Falange & media & Falange & distal \\
\hline edades & Mano der & Mano izq & Mano der & Mano izq & Mano der & Mano izq \\
\hline $18-19$ & $46 \pm 14$ & $46 \pm 14$ & $32 \pm 8$ & $32 \pm 7$ & $23 \pm 4$ & $23 \pm 4$ \\
\hline $20-24$ & $49 \pm 13$ & $48 \pm 13$ & $32 \pm 6$ & $32 \pm 6$ & $23 \pm 4$ & $23 \pm 4$ \\
\hline $25-29$ & $50 \pm 12$ & $49 \pm 12$ & $33 \pm 5$ & $32 \pm 6$ & $23 \pm 2$ & $24 \pm 2$ \\
\hline $30-34$ & $39 \pm 17$ & $38 \pm 16$ & $28 \pm 7$ & $28 \pm 8$ & $23 \pm 9$ & $23 \pm 9$ \\
\hline $35-39$ & $50 \pm 12$ & $50 \pm 11$ & $32 \pm 7$ & $32 \pm 7$ & $23 \pm 4$ & $22 \pm 4$ \\
\hline $40-44$ & $49 \pm 11$ & $48 \pm 12$ & $32 \pm 7$ & $32 \pm 6$ & $24 \pm 2$ & $24 \pm 2$ \\
\hline $45-49$ & $49 \pm 12$ & $49 \pm 12$ & $33 \pm 6$ & $32 \pm 6$ & $24 \pm 3$ & $23 \pm 3$ \\
\hline $50-54$ & $46 \pm 14$ & $45 \pm 14$ & $31 \pm 6$ & $31 \pm 7$ & $24 \pm 3$ & $23 \pm 3$ \\
\hline $55-59$ & $41 \pm 16$ & $41 \pm 16$ & $29 \pm 9$ & $28 \pm 8$ & $23 \pm 4$ & $23 \pm 4$ \\
\hline $60-64$ & $40 \pm 15$ & $37 \pm 14$ & $28 \pm 8$ & $29 \pm 8$ & $23 \pm 3$ & $22 \pm 2$ \\
\hline $65-69$ & $47 \pm 18$ & $46 \pm 19$ & $32 \pm 10$ & $32 \pm 10$ & $23 \pm 3$ & $24 \pm 3$ \\
\hline
\end{tabular}


Tabla VIII. Longitudes promedio del sector de las falanges del dedo medio de hombres, expresado en mm.

\begin{tabular}{|c|c|c|c|c|c|c|}
\hline & Falange & proximal & Falange & media & Falange & distal \\
\hline edades & Mano der & Mano izq & Mano der & Mano izq & Mano der & Mano izq \\
\hline $18-19$ & $55 \pm 13$ & $53 \pm 12$ & $35 \pm 6$ & $35 \pm 6$ & $25 \pm 4$ & $25 \pm 4$ \\
\hline $20-24$ & $53 \pm 13$ & $54 \pm 13$ & $36 \pm 6$ & $35 \pm 6$ & $25 \pm 3$ & $25 \pm 3$ \\
\hline $25-29$ & $56 \pm 14$ & $55 \pm 14$ & $37 \pm 8$ & $36 \pm 8$ & $27 \pm 4$ & $27 \pm 4$ \\
\hline $30-34$ & $53 \pm 13$ & $53 \pm 12$ & $35 \pm 7$ & $36 \pm 7$ & $27 \pm 4$ & $26 \pm 4$ \\
\hline $35-39$ & $47 \pm 16$ & $47 \pm 14$ & $33 \pm 8$ & $32 \pm 7$ & $26 \pm 3$ & $26 \pm 3$ \\
\hline $40-44$ & $55 \pm 16$ & $55 \pm 16$ & $34 \pm 11$ & $35 \pm 11$ & $26 \pm 6$ & $26 \pm 7$ \\
\hline $45-49$ & $57 \pm 13$ & $57 \pm 13$ & $38 \pm 8$ & $37 \pm 8$ & $26 \pm 3$ & $26 \pm 2$ \\
\hline $50-54$ & $56 \pm 13$ & $56 \pm 12$ & $36 \pm 7$ & $36 \pm 7$ & $26 \pm 4$ & $26 \pm 4$ \\
\hline $55-59$ & $53 \pm 17$ & $52 \pm 16$ & $36 \pm 10$ & $35 \pm 9$ & $27 \pm 4$ & $28 \pm 5$ \\
\hline $60-64$ & $49 \pm 18$ & $51 \pm 17$ & $35 \pm 10$ & $35 \pm 8$ & $26 \pm 4$ & $26 \pm 4$ \\
\hline
\end{tabular}

Tabla IX. Longitudes promedio del sector de las falanges del dedo anular de mujeres, expresado en mm.

\begin{tabular}{|c|c|c|c|c|c|c|}
\hline & Falange & proximal & Falange & media & Falange & distal \\
\hline edades & Mano der & Mano izq & Mano der & Mano izq & Mano der & Mano izq \\
\hline $18-19$ & $51 \pm 12$ & $50 \pm 11$ & $34 \pm 6$ & $34 \pm 5$ & $24 \pm 4$ & $25 \pm 3$ \\
\hline $20-24$ & $50 \pm 13$ & $50 \pm 12$ & $34 \pm 6$ & $34 \pm 5$ & $25 \pm 3$ & $25 \pm 3$ \\
\hline $25-29$ & $51 \pm 13$ & $52 \pm 14$ & $35 \pm 8$ & $35 \pm 7$ & $27 \pm 4$ & $27 \pm 4$ \\
\hline $30-34$ & $49 \pm 12$ & $49 \pm 11$ & $34 \pm 7$ & $35 \pm 6$ & $27 \pm 4$ & $26 \pm 4$ \\
\hline $35-39$ & $44 \pm 15$ & $44 \pm 14$ & $31 \pm 8$ & $30 \pm 8$ & $26 \pm 4$ & $26 \pm 6$ \\
\hline $40-44$ & $52 \pm 15$ & $51 \pm 13$ & $33 \pm 9$ & $33 \pm 11$ & $25 \pm 6$ & $25 \pm 7$ \\
\hline $45-49$ & $52 \pm 12$ & $52 \pm 12$ & $36 \pm 7$ & $36 \pm 8$ & $26 \pm 4$ & $26 \pm 3$ \\
\hline $50-54$ & $52 \pm 12$ & $52 \pm 12$ & $34 \pm 6$ & $34 \pm 6$ & $25 \pm 3$ & $25 \pm 3$ \\
\hline $55-59$ & $49 \pm 17$ & $49 \pm 16$ & $33 \pm 9$ & $33 \pm 9$ & $27 \pm 3$ & $26 \pm 4$ \\
\hline $60-64$ & $45 \pm 17$ & $46 \pm 16$ & $33 \pm 9$ & $33 \pm 8$ & $27 \pm 4$ & $26 \pm 4$ \\
\hline
\end{tabular}

Tabla X. Longitudes promedio del sector de las falanges del dedo anular de hombres, expresado en mm.

\begin{tabular}{|c|c|c|c|c|c|c|}
\hline & Falange & proximal & Falange & media & Fal ange & distal \\
\hline edades & Mano der & Mano izq & Mano der & Mano izq & Mano der & Mano izq \\
\hline $18-19$ & $44 \pm 13$ & $43 \pm 13$ & $31 \pm 6$ & $31 \pm 6$ & $23 \pm 3$ & $23 \pm 3$ \\
\hline $20-24$ & $45 \pm 12$ & $45 \pm 11$ & $30 \pm 6$ & $30 \pm 6$ & $23 \pm 4$ & $23 \pm 4$ \\
\hline $25-29$ & $45 \pm 12$ & $45 \pm 12$ & $31 \pm 6$ & $32 \pm 7$ & $23 \pm 3$ & $23 \pm 2$ \\
\hline $30-34$ & $35 \pm 16$ & $34 \pm 14$ & $26 \pm 8$ & $24 \pm 8$ & $21 \pm 4$ & $22 \pm 4$ \\
\hline $35-39$ & $47 \pm 10$ & $46 \pm 11$ & $30 \pm 7$ & $30 \pm 7$ & $22 \pm 4$ & $22 \pm 4$ \\
\hline $40-44$ & $46 \pm 11$ & $46 \pm 10$ & $31 \pm 6$ & $31 \pm 6$ & $24 \pm 2$ & $24 \pm 3$ \\
\hline $45-49$ & $45 \pm 11$ & $45 \pm 11$ & $31 \pm 6$ & $30 \pm 6$ & $23 \pm 3$ & $23 \pm 3$ \\
\hline $50-54$ & $43 \pm 13$ & $42 \pm 13$ & $30 \pm 7$ & $30 \pm 6$ & $23 \pm 3$ & $23 \pm 3$ \\
\hline $55-59$ & $37 \pm 15$ & $37 \pm 15$ & $27 \pm 8$ & $27 \pm 8$ & $23 \pm 4$ & $23 \pm 4$ \\
\hline $60-64$ & $34 \pm 14$ & $35 \pm 13$ & $27 \pm 7$ & $28 \pm 8$ & $22 \pm 2$ & $22 \pm 3$ \\
\hline $65-69$ & $42 \pm 19$ & $42 \pm 18$ & $30 \pm 9$ & $31 \pm 10$ & $24 \pm 1$ & $24 \pm 1$ \\
\hline
\end{tabular}


En la biometría especifica de la mano, no hubo diferencia significativa entre la lateralidad (mano derecha e izquierda) de los individuos en las diferentes edades y gene- ro. Existen diferencias significativas en el largo y ancho de la mano y longitud del dedo medio, al realizar las comparaciones entre género, edad y estatura.

Tabla XI. Longitudes promedio del sector de las falanges del dedo mínimo de mujeres, expresado en mm.

\begin{tabular}{|c|c|c|c|c|c|c|}
\hline & Falange & proximal & Falange & media & Falange & distal \\
\hline edades & Mano der & Mano izq & Mano der & Mano izq & Mano der & Mano izq \\
\hline $18-19$ & $40 \pm 9$ & $40 \pm 9$ & $25 \pm 5$ & $25 \pm 5$ & $22 \pm 3$ & $23 \pm 3$ \\
\hline $20-24$ & $40 \pm 10$ & $41 \pm 10$ & $25 \pm 6$ & $25 \pm 5$ & $23 \pm 3$ & $23 \pm 3$ \\
\hline $25-29$ & $42 \pm 12$ & $42 \pm 12$ & $27 \pm 6$ & $28 \pm 8$ & $24 \pm 3$ & $24 \pm 3$ \\
\hline $30-34$ & $40 \pm 10$ & $40 \pm 10$ & $26 \pm 6$ & $26 \pm 5$ & $23 \pm 3$ & $23 \pm 3$ \\
\hline $35-39$ & $34 \pm 13$ & $35 \pm 12$ & $24 \pm 7$ & $23 \pm 7$ & $23 \pm 3$ & $23 \pm 3$ \\
\hline $40-44$ & $40 \pm 15$ & $39 \pm 15$ & $27 \pm 7$ & $26 \pm 7$ & $22 \pm 6$ & $22 \pm 5$ \\
\hline $45-49$ & $43 \pm 10$ & $43 \pm 11$ & $27 \pm 5$ & $27 \pm 7$ & $23 \pm 4$ & $23 \pm 3$ \\
\hline $50-54$ & $43 \pm 10$ & $42 \pm 10$ & $26 \pm 5$ & $25 \pm 5$ & $22 \pm 3$ & $22 \pm 3$ \\
\hline $55-59$ & $39 \pm 14$ & $38 \pm 13$ & $25 \pm 7$ & $25 \pm 8$ & $24 \pm 4$ & $23 \pm 3$ \\
\hline $60-64$ & $38 \pm 14$ & $38 \pm 14$ & $25 \pm 8$ & $26 \pm 7$ & $23 \pm 4$ & $24 \pm 3$ \\
\hline
\end{tabular}

Tabla XII. Longitudes promedio del sector de las falanges del dedo mínimo de hombres, expresado en mm.

\begin{tabular}{|c|c|c|c|c|c|c|}
\hline & Falange & proximal & Falange & media & Falange & distal \\
\hline edades & Mano der & Mano izq & Mano der & Mano izq & Mano der & Mano izo \\
\hline $18-19$ & $36 \pm 10$ & $35 \pm 11$ & $22 \pm 5$ & $23 \pm 5$ & $20 \pm 3$ & $21 \pm 3$ \\
\hline $20-24$ & $37 \pm 9$ & $36 \pm 10$ & $22 \pm 5$ & $22 \pm 5$ & $20 \pm 3$ & $20 \pm 3$ \\
\hline $25-29$ & $37 \pm 9$ & $37 \pm 10$ & $23 \pm 5$ & $23 \pm 3$ & $20 \pm 2$ & $20 \pm 2$ \\
\hline $30-34$ & $29 \pm 14$ & $29 \pm 13$ & $19 \pm 6$ & $19 \pm 7$ & $19 \pm 3$ & $19 \pm 3$ \\
\hline $35-39$ & $37 \pm 10$ & $37 \pm 9$ & $22 \pm 5$ & $22 \pm 6$ & $20 \pm 3$ & $19 \pm 3$ \\
\hline $40-44$ & $37 \pm 9$ & $37 \pm 9$ & $23 \pm 5$ & $22 \pm 6$ & $20 \pm 3$ & $20 \pm 3$ \\
\hline $45-49$ & $37 \pm 9$ & $37 \pm 10$ & $23 \pm 4$ & $23 \pm 5$ & $20 \pm 3$ & $20 \pm 3$ \\
\hline $50-54$ & $34 \pm 12$ & $33 \pm 11$ & $21 \pm 6$ & $22 \pm 6$ & $20 \pm 3$ & $20 \pm 2$ \\
\hline $55-59$ & $30 \pm 13$ & $30 \pm 13$ & $20 \pm 7$ & $20 \pm 7$ & $19 \pm 4$ & $20 \pm 3$ \\
\hline $60-64$ & $30 \pm 12$ & $29 \pm 10$ & $20 \pm 6$ & $21 \pm 5$ & $19 \pm 2$ & $20 \pm 3$ \\
\hline $65-69$ & $36 \pm 15$ & $36 \pm 15$ & $23 \pm 7$ & $23 \pm 7$ & $20 \pm 5$ & $20 \pm 3$ \\
\hline
\end{tabular}

\section{DISCUSIÓN}

La mano se expone diariamente a diversas actividades que pueden a veces, conllevar riesgos de lesiones, que pueden impedir sus capacidades funcionales, parcial o totalmente. Es conocida la frecuencia de fracturas en la región de la muñeca y mano, de tal modo que los dedos pueden ser seriamente afectados. En una descripción de Flynn (1982) sobre fracturas de los metacarpianos y falanges, señaló que la falange distal era la más afectada $(45 \%)$, siguiendo los metacarpianos (32\%) y las falanges proximales $(17,3 \%)$. Por otra parte, el uso constante de teclados de computadores y máquinas operadas manualmente pueden llevar a una serie de lesiones laborales, tales como tendinitis, bursitis, síndrome del canal del carpo, artritis, artrosis, etc., entidades clínicas que hacen que la mano pierda gran parte de su eficacia. De ahí, que resulta de gran interés conocer con detalles las estructuras que le componen, desde el punto de vista anatómico y biométrico. 
La literatura muestra numerosos estudios sobre esta importante parte del cuerpo, los que tienen una gran utilidad para la cirugía reparadora de la misma. Sin embargo, estudios biométricos han sido poco desarrollados y más aún, en nuestra población, de ahí que resulta de interés conocer diversos parámetros de la mano, aportando conocimientos que puedan ser de utilidad en los tratamientos reparadores frente a lesiones que incapacitan parcial o totalmente a ésta. Por otra parte, el conocer la mano y sus componentes, tejidos duros y blandos, permite a los fabricantes de diversos utensilios para la vida laboral, confeccionarlos de acuerdo al tamaño de la mano y de los dedos, por ejemplo, guantes, herramientas y otros.

De los datos obtenidos se puede mostrar que el sector de la falange proximal del dedo medio es el de mayor longitud, en ambos sexos, siendo el de menor longitud el sector correspondiente al dedo mínimo. Los sectores correspondientes a las falanges proximales de los dedos anular e indicador son aproximadamente iguales, menores al del dedo medio, concordando con lo expresado por Kaplan (1965). Con respecto al sector de las falanges medias, el de mayor longitud corresponde al dedo medio, seguido del dedo anular y del dedo indicador, en orden decreciente, siendo el sector más corto, el del dedo mínimo.

Los sectores de las falanges distales tienen leves diferencias, siendo mayor el del dedo medio, siguiendo el del dedo anular que es aproximadamente 0,2 a $0,4 \mathrm{~mm}$ menor; el sector de la falange distal del dedo indicador es menor al del dedo anular en aproximadamente 1,2 a 1,5 mm, siendo el más corto, el correspondiente al dedo mínimo. Estos resultados son similares a los descritos por Kaplan. Algunos textos clásicos son poco descriptivos respecto a estas variables, indicando apenas que las falanges como huesos, no como sector con tejidos blandos, son de cortas dimensiones (Testut \& Latarjet, 1969) o simplemente no tienen información sobre el tema (Williams et al., 1995).

Considerando los promedios de las longitudes de los dedos de la mano de nuestra población, tanto en hombres como en mujeres, el dedo medio es el más largo y el pulgar el más corto, siendo el indicador levemente menor que el anular y el mínimo menor que el indicador y anular.

Algunos estudios sobre la longitud y diámetro de los dedos se han llevado a cabo con el propósito de aportar al mejoramiento de ciertos mecanismos del cierre de vidrios en automóviles, para así evitar lesiones en los dedos, especialmente de niños, como es el caso de la investigación llevada a cabo por Hohendorff et al. (2010) en 160 niños de 3 a 10 años de edad, quienes obtuvieron valores con los que proporcionalmente concordamos de acuerdo al párrafo an- terior, señalando también que todas las dimensiones se incrementaron con la edad. En las edades estudiadas no encontraron diferencias entre sexos para longitud y diámetro. En nuestro estudio, hubo diferencias entre sexos y la estatura para el caso del dedo medio.

La longitud de las falanges ha sido investigada por autores como Aydinlioglu et al. (1998) y Case \& Ross (2006), y al sumar esas longitudes, el resultado fue algo diferente con respecto al obtenido en dedos de individuos en que se ha considerado sus componentes blandos, como es el caso de nuestro estudio. Tanto los registros radiográficos como aquellos obtenidos de esqueletos son menores a los datos de nuestro estudio y al comparar los resultados entre los autores mencionados los obtenidos de esqueletos son algo mayores a los radiográficos, considerando que las manos eran de diferentes grupos étnicos. En las muestras de Aydinlioglu et al. las dos falanges del pulgar daban una longitud de $54 \mathrm{~mm}$ en el hombre y de 48 $\mathrm{mm}$ en la mujer; las tres falanges del dedo medio tenían una longitud de $94 \mathrm{~mm}$ en el hombre y $86 \mathrm{~mm}$ en la mujer; las del dedo mínimo tenían 71 y $63 \mathrm{~mm}$, respectivamente, en cambio, en las de Case \& Ross, para las dos falanges del pulgar se obtuvo un promedio de $57,3 \mathrm{~mm}$ y $50,2 \mathrm{~mm}$; las tres del dedo medio 97,4 y $87,4 \mathrm{~mm}$ y las del dedo mínimo 74,1 y $65 \mathrm{~mm}$, respectivamente.

Recientemente, Eshak et al. (2011) estudiando por tomografía computarizada a 122 individuos egipcios registraron las longitudes de las falanges, cuyos valores fueron los siguientes: las dos falanges del pulgar daban una longitud de $51,8 \mathrm{~mm}$ en el hombre y de $47,2 \mathrm{~mm}$ en la mujer; las tres falanges del dedo medio tenían una longitud de 89,4 $\mathrm{mm}$ en el sexo masculino y $81 \mathrm{~mm}$ en el femenino; las del dedo mínimo tenían 69,7 y $61,9 \mathrm{~mm}$, respectivamente, pudiendo apreciar que estos valores son menores a los encontrados por los autores previamente mencionados y a nuestro propios valores, haciendo hincapié que las mediciones registradas en nuestro estudio incorporaron los componentes blandos de los dedos.

No encontramos datos bibliográficos referentes a la biometría de los componentes de la mano en nuestra población, excepto un trabajo realizado por Garrido-Varas \& Thompson (2011) en las falanges proximales de muestras de dos sitios arqueológicos de Atacama, donde encontró que la mayor de ellas correspondía a la del dedo medio con un promedio de $42 \mathrm{~mm}+2,4$ y la menor la del dedo pulgar con un promedio de $28 \mathrm{~mm}+1,86$; la del dedo anular tuvo un valor de $39,8 \mathrm{~mm}$. En ambos grupos arqueológicos los resultados fueron similares y comparándolos con un grupo de individuos de la población actual de Inglaterra, los valores registrados en estos últimos fueron mayores. 
El tamaño de la palma y de la mano en general, es diferente si consideramos el sexo, edad y estatura, ya que al realizar las pruebas estadísticas se obtuvo diferencias significativas en el largo y ancho de la mano y longitud del dedo medio, al realizar las comparaciones entre estos grupos.

Los datos aportados en este trabajo son de relevante importancia, ya que permiten tener un conocimiento real de variables biométricas de la mano en individuos chilenos, los que pueden ser utilizados como base para la confección de diversos utensilios usados a diario por las personas en actividades laborales, así como también ser información para los profesionales que actúan en el área quirúrgica y de restauración de la funcionalidad de esta importante parte del cuerpo.

AGRADECIMIENTOS: Dirección Investigación e Innovación (DII) de la Pontificia Universidad Católica de Valparaíso, Chile, por su constante apoyo.

BINVignAT, O; ALMAgià, A.; LIZANA, P. \& OLAVE, E. Biometric aspects of the hand in Chilean individuals. Int. J. Morphol., 30(2):599-606, 2012.

SUMMARY: The hand is essential to perform various actions both in the workplace and in entertainment and sports, may perform other functions from very basic to highly specialized. The hand function can be analyzed best when it is divided into parts, although it is somewhat artificial and merely didactic, allowing a better understanding of the labor activity as a unit. Because of the importance of this part of the body and biometric parameters limited information that exists in our population, it has become necessary to a thorough study on the dimensions of the hand as a whole, as well as its components, the fingers. Accordingly, we analyzed 1,196 hands belonging to people of both sexes (267 men and 331 women) aged between 18 and 65, born and living in VII Maule Region of Chile. Each patient underwent an anthropometric examination of general and specific one hand. The variables considered were: hand length, hand width, finger length, length of the phalanges sector. The variables were studied according to sex, age and sides. Part of the results were as follows: in males, the average length of the right hand was $184.9 \mathrm{~mm}$ and the left of 185.6; in females was $167.6 \mathrm{~mm}$ on the right side and of 168.9 on the left. With respect to the length of the fingers in males, whereas the sum of the averages for each sector of the corresponding phalanges, the averages were: thumb, $72.5 \mathrm{~mm}$ in the right hand and $72.4 \mathrm{~mm}$ in the left; index finger, 104.2 and $104.5 \mathrm{~mm}$; middle finger, 115 and $114.6 \mathrm{~mm}$; ring finger, $109.1 \mathrm{and} 108.9 \mathrm{~mm}$; little finger, 88.5 and $88.4 \mathrm{~mm}$ respectively. Among females, the average lengths were: right thumb, $63.5 \mathrm{~mm}$ and $62.6 \mathrm{~mm}$ in the left; index, 91 and $90.7 \mathrm{~mm}$; middle finger, 100.4 and $99.2 \mathrm{~mm}$; ring finger, 94.4 and $94.2 \mathrm{~mm}$ and the little finger, 75.8 and $75.9 \mathrm{~mm}$, respectively. Data reported in this study are important because they allow a real knowledge of biometric variables in Chilean hands.

KEY WORDS: Hand; Fingers; Phalanges; Biometry.

\section{REFERENCIAS BIBLIOGRÁFICAS}

Aydinlioglu, A.; Akpinar, F. \& Tosun, N. Mathematical relations between the lengths of the metacarpal bones and phalanges: Surgical significance. Tohoku J. Exp.Med., 185: 209-16, 1998.

Case, T. \& Ross, A. Sex determination from hand and foot bone lengths. J. Forensic Sci.. 52(2): 264-70, 2007.

Eshak, G.; Ahmed, H. \& Abdel Gawad, E. Gender determination from hand bones length and volume using multidetector computed tomography: A study in Egyptian people. J. Forensic and Legal Med., 18:246-252, 2011.

Flynn, E. Hand Surgery. 3rd Ed., Baltimore, Williams \& Wilkins, 1982.

Garrido- Varas, C.E. \& Thompson, T.J.U. Metric dimensions of the proximal phalanges of the human hand and their relationship to side, position, and asymmetry. J. Comparative Human Biol., 62:126-143, 2011.

Hohendorff, B.; Weidermann, C.; Burkhart, K.J.; Rommens, P.M.; Prommersberger, K.J. \& Konerding, M.A. Lengths, girths, and diameters of children's fingers from 3 to 10 years of age. Ann. Anat., 192:156-61, 2010.
Kaplan, E. B. Functional and Surgical Anatomy of the Hand. 2nd Ed. Philadelphia, Lippincott Co., 1965.

Testut, L. \& Latarjet, A. Tratado de Anatomía Humana. Barcelona, Salvat, 1969. V. 3.

Williams, R L.; Warwick, R.; Dyson, M. \& Bannister, L. H. Gray Anatomía. 37a Ed. Rio de Janeiro, Guanabara-Koogan, 1995. V. 2.

Dirección para correspondencia:

Prof. Dr. Octavio Binvignat Gutiérrez

Universidad Autónoma de Chile

Talca - CHILE

Email: obinvignatg@uautonoma.cl

Recibido : 07-01-2012

Aceptado: 02-03-2012 